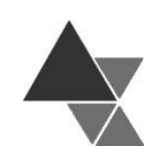

\title{
Adaptações do leite em mães de recém-nascidos prematuros
}

\author{
Emerson Pinheiro Ferreira ${ }^{1}$ e Cesar Henrique de Carvalho Moraes ${ }^{2}$
}

Apesar da importância de proteínas (PTN), lipídios (LIP) e Cálcio (Ca) no desenvolvimento de recém-nascidos algumas lacunas acerca das possíveis diferenças no leite de genitoras a termo (LMAT) comparado ao leite de genitoras pré-termo (LMPT) precisam ser elucidadas. É objetivo deste estudo caracterizar adaptações fisiológicas e nutricionais comparando o LMAT com o LMPT, especificamente em relação às PTN, LIP ou Ca. Para tal, foi realizada uma revisão narrativa nas bases Google Acadêmico e PubMed, no período de 2004 a 2020, com idiomas em português e inglês. Foram critérios de inclusão: artigos e teses que discutiam a composição nutricional do leite considerando teor de LIP, PTN ou Ca. Para a realização da pesquisa os seguintes termos livres foram relacionados: Leite materno, pré-termo, a termo, proteína, lipídeos e cálcio. Em conclusão, o conjunto de estudos demonstra haver variação no conteúdo de PTN e LIP quando se compara o LMPT ao LMAT, o que ocorre possivelmente pela necessidade diferenciada dos bebês para cada situação. Adicionalmente, verifica-se carência de Ca no LMPT, o que não atende as necessidades nutricionais de prematuros. Estes dados permitem a particularização para o aleitamento materno exclusivo e a compreensão das especificidades fisiológicas e nutricionais nestas diferentes condições de gestação.

Palavras-chave: Leite materno; Pré-termo; A termo; Proteína; Lipídeos; Cálcio.

\section{Adaptations of milk in mothers of premature newborns}

Despite the importance of proteins (PTN), lipids (LIP) and calcium (Ca) in the development of newborns, some gaps about the possible differences in the milk of full-term mothers (LMAT) compared to milk of preterm mothers (LMPT) need to be clarified. The objective of this study is to characterize the physiological and nutritional adaptations by comparing the LMAT with the LMPT, specifically in relation to PTN, LIP or Ca content. For this purpose, a narrative review was performed on Google Academic and PubMed, from 2004 to 2020, regarding languages in Portuguese and English. Inclusion criteria were articles and theses that discussed the nutritional composition of milk considering LIP, PTN or Ca content. The following free terms were correlated to the research: breast milk, preterm, term, protein, lipids and calcium. In conclusion, the set of studies shows that there is variation in the content of PTN and LIP when comparing LMPT to LMAT, which occurs possibly due to the different needs of babies for each situation. Additionally, there is a lack of Ca in LMPT which does not meet the nutritional needs of premature infants. These data allow the particularization for exclusive breastfeeding and the understanding of the physiological and nutritional specificities in these different gestation conditions.

Keywords: Breast milk; Preterm; Term; Protein; Lipids; Calcium.

\footnotetext{
${ }^{1}$ Especializado em Nutrição em Saúde Cardiovascular. Instituto do Coração (InCor), Universidade de São Paulo, Faculdade de Medicina, Brasil. E-mail: emersonpinheirof4@gmail.com. ID ORCID: https://orcid.org/0000-0002-5870-4014. Endereço para Correspondência: Lipid Clinic Heart Institute (InCor), Hospital da Faculdade de Medicina da Universidade de São Paulo, São Paulo (SP) Brasil. Avenida Dr. Enéas de Carvalho Aguiar, no 44, CEP 05403-000, SP, Brasil. Tel: 01126615320 / 01126615299.

${ }^{2}$ Faculdade de Saúde Pública/USP e Docente da Universidade Paulista (UNIP). ID ORCID: https://orcid.org/0000-0003-1108-8100. E-mail: cesar.moraes@usp.br
} 


\section{INTRODUÇÃO}

O leite materno (LM) é um agente natural e biologicamente adequado ao neonato devido ao seu conteúdo nutricional, bioativos e imunomodulador que auxilia na recuperação, sobrevivência e desenvolvimento do bebê ${ }^{[1]}$. Consiste no alimento ideal para o desenvolvimento de lactentes durante os seis primeiros meses de vida e sua recomendação torna-se fundamental na promoção da saúde, diminuindo os indicadores de morbimortalidade infantil[2]. Definem-se "a termo" (AT) as gestações de 37 a 42 semanas e "pré-termo" (PT) para a gestação com menos de 37 semanas[ ${ }^{[3]}$, sendo a prematuridade um dos maiores riscos à saúde humana e uma das principais causas de morbimortalidade neonatal[4].

A oferta exclusiva de leite de genitoras de pré-termos (LMPT) para seus bebês é extremamente essencial, pois diminui substancialmente a incidência ou magnitude de algumas patologias inerentes a essa fase da vida, como diarreia, infecções e alergias ${ }^{[5]}$. Além disso, prematuros alimentados com o leite da própria mãe apresentam potencial maior de ganho de peso e vantagens no crescimento linear e cerebral[6].

Segundo Moura[7], a constituição do LM sofre modificações em sua composição de acordo com o tempo de amamentação e período do dia a qual o leite é ofertado ao bebê. Tais modificações pressupõem uma adequação às necessidades fisiológicas e nutricionais do recém-nascido[ [7]. Um estudo realizado por Gidrewicz e Fenton ${ }^{[8]}$ verificou uma variação nutricional do LM, com alternância entre o conteúdo de proteína (PTN) e lipídeo (LIP), sendo a idade pós-natal e os estágios gestacionais maternos, importantes preditores destas mudanças. Já Sabatier et al.[9], observaram no LM uma tendência crescente nas concentrações do fósforo $(\mathrm{P})$ do LM durante a primeira semana pós-parto, com diminuição gradual durante a lactação. As concentrações de cálcio $(\mathrm{Ca})$ foram mais altas na primeira semana após o nascimento e diminuíram gradualmente durante a lactação.
Apesar da importância dos nutrientes como PTN, LIP e do Ca no desenvolvimento humano e das vantagens fisiológicas da captação inicial desses elementos a partir do LM, não há consenso na literatura acerca das possíveis diferenças em relação a PTN, LIP e do Ca, no LMPT quando comparado ao leite de genitoras a termo (LMAT). A consideração deste conhecimento será de fundamental importância para a individualização da prevenção e promoção da saúde da gestante ${ }^{[10]}$ em suas diferentes condições: a termo e pré-termo. Portanto, considerar estas distinções torna-se viável, ponderando práticas educativas específicas para os diferentes perfis de gestantes. Assim, garante-se que o aleitamento materno exclusivo seja mantido ou predomine independentemente da condição em que se encontre a genitora.

Esse trabalho tem como objetivo descrever por meio dos dados existentes na literatura as diferenças nutricionais do LMPT versus LMAT, especificamente em relação às PTN, LIP e Ca.

\section{REVISÃO DA BIBLIOGRAFIA}

Trata-se de estudo de carácter exploratório, realizado por meio de revisão narrativa, na qual foram consultados artigos encontrados nas bases de dados Google Acadêmico e PubMed, publicados entre o período de 2004 a 2020, com idiomas em português e inglês. Adicionalmente, alguns artigos de anos anteriores foram mencionados ao decorrer do trabalho, devido a sua importância sobre o assunto.

Para a realização da pesquisa foram relacionados os termos livres: leite materno, pré-termo, a termo, proteína, lipídeos e cálcio. Foram selecionados 24 trabalhos considerando-se como critério de inclusão a discussão em alguma medida sobre a composição nutricional do leite englobando o teor de PTN, LIP ou $\mathrm{Ca}$, sendo que dez artigos analisaram conjuntamente estes macronutrientes e micronutrientes e, portanto, foram elegíveis para o escopo central desta revisão (Quadro 1). 
Ressalta-se que os artigos escolhidos para o escopo central desta revisão foram analisados de maneira qualitativa, já que as unidades de grandeza reportadas para a descrição dos resultados dos nutrientes variaram entre os estudos, o que dificultou uma análise quantitativa comum.

\section{PROTEÍNAS}

Concentrações maiores de PTN foram encontradas por Gross et al.[11] no LMPT comparados aos LMAT $(p<0,005)$, atingindo a maior quantidade de PTN no 3o dia pós-parto e concentrações médias no 28 o pós-parto. $\mathrm{O}$ nitrogênio não proteico esteve em torno de $15 \%$ no LMAT e 19\% no LMPT. Os autores concluíram que, de alguma forma, o LMPT aproximou-se mais das necessidades nutricionais do bebê prematuro, mas sem explicação aparente das motivações para tal. 
Quadro 1 - Relação de proteínas, lipídeos e cálcio no leite de lactantes a termos e pré-termos.

\begin{tabular}{|c|c|c|c|c|c|c|c|}
\hline \multirow{2}{*}{ Autor, ano e país } & \multirow{2}{*}{ Objetivo } & \multirow{2}{*}{ Amostra } & \multicolumn{4}{|c|}{ Resultados (Média/DP) } & \multirow{2}{*}{ Conclusão } \\
\hline & & & PTN & LIP & $\mathrm{Ca}$ & $\mathbf{P}$ & \\
\hline \multirow[b]{2}{*}{$\begin{array}{l}\text { Hsu et al., 2014[14]. } \\
\text { Taiwan. }\end{array}$} & \multirow[b]{2}{*}{$\begin{array}{c}\text { Investigar as } \\
\text { mudanças na } \\
\text { composição do } \\
\text { LMPT (IG }<35 \\
\text { SMN) durante as } \\
\text { primeiras semanas } \\
\text { de lactação. }\end{array}$} & \multirow[b]{2}{*}{$\begin{array}{c}\text { Coleta: } 1 \text { a semana } \\
\mathbf{n}=17 \\
(\mathrm{IG}<35 \mathrm{SMN}) \\
\mathbf{n}=15 \\
(\mathrm{IG} \geq 37 \mathrm{SMN})\end{array}$} & \multicolumn{2}{|c|}{$\mathrm{g} / \mathrm{dl}$} & \multicolumn{2}{|c|}{$\mathrm{mg} / \mathrm{l}$} & \multirow[b]{2}{*}{$\begin{array}{l}\text { Houve diferenças na composição de nutrientes durante os } \\
\text { períodos, com maior concentração de LIP }(p=0,001) \text { e P ( } p \\
\qquad=0,022) \text { e menor na PTN }(p=0,000) . \\
\text { A quantificação de P no LMPT foi menor que a normal, } \\
\text { sugerindo que pode ser indicada uma monitorização } \\
\text { cuidadosa da massa óssea do corpo. }\end{array}$} \\
\hline & & & $\begin{array}{c}\text { PT: } 1,685 \\
(\mathrm{DP} \pm 0.46) \\
\\
\text { AT: } 1,567 \\
(\mathrm{DP} \pm 0.32)\end{array}$ & $\begin{array}{l}\text { PT: } 2,650 \\
(\mathrm{DP} \pm 072) \\
\\
\text { AT: } 2,757 \\
(\mathrm{DP} \pm 0.32)\end{array}$ & $\begin{array}{l}\text { PT: } 31,25 \\
\text { (DP } \pm 8.33 \text { ) } \\
\\
\text { AT: } 31,54 \\
\text { (DP } \pm 8,82 \text { ) }\end{array}$ & $\begin{array}{l}\text { PT: } 4,304 \\
(\mathrm{DP} \pm 3,06) \\
\\
\text { AT: } 5,400 \\
\text { (DP } \pm 2,65)\end{array}$ & \\
\hline \multirow[b]{2}{*}{$\begin{array}{c}\text { Bauer e Gerss, 2010[33]. } \\
\text { Alemanha. }\end{array}$} & \multirow[b]{2}{*}{$\begin{array}{c}\text { Investigar a } \\
\text { composição do leite } \\
\text { de lactantes com IG } \\
<28 \text { semanas } \\
\text { comparado a outras } \\
\text { IG. }\end{array}$} & \multirow[b]{2}{*}{$\begin{array}{c}\text { Coleta: } 8 \text { semanas } \\
\mathbf{n}=102 \\
(\text { IG } 23-33 \mathrm{SMN}) \\
\mathbf{n}=10 \\
(>37 \mathrm{SMN})\end{array}$} & \multicolumn{2}{|c|}{$\mathrm{g} / \mathrm{dl}$} & \multicolumn{2}{|c|}{$\mathrm{Mmol} / \mathrm{dl}$} & \multirow[b]{2}{*}{$\begin{array}{l}\text { As concentrações de LIP foram significativamente maiores } \\
\text { no leite prematuro que no AT }(p<0,05) \text {. Não foram } \\
\text { observadas diferenças entre os grupos prematuros. } \\
\text { O teor de PTN no leite extremamente prematuro }(<28) \text { foi } \\
\text { significativamente maior do que no leite moderadamente } \\
\text { prematuro e LMAT ( } p<0,001) \text {. } \\
\text { Não foram estabelecidas diferenças nas concentrações de } \\
\text { fosfato e Ca entre os diferentes grupos de LMPT e o } \\
\text { LMAT. }\end{array}$} \\
\hline & & & $\begin{array}{c}\text { PT: } \\
(\mathrm{IG}<28): 2,3 \\
(\mathrm{DP} \pm 0,5) \\
\text { PT: } \\
(\text { IG 28-31): } 2,1 \\
\text { (DP } \pm 0,3) \\
\text { PT: } \\
\text { (IG 32-33): } 1,9 \\
\text { (DP } \pm 0,3) \\
\text { AT: } 1,6 \\
\text { (DP } \pm 0,4)\end{array}$ & $\begin{array}{c}\text { PT: } \\
\text { (IG <28): 4,4 } \\
\text { (DP } \pm 0,9) \\
\text { PT: } \\
(\text { IG 28-31): 4,4 } \\
\text { (DP } \pm 0,8) \\
\text { PT: } \\
\text { (IG 32-33): 4,8 } \\
\text { (DP } \pm 1,0) \\
\text { AT: 4,1 } \\
\text { (DP } \pm 0,7)\end{array}$ & $\begin{array}{c}\text { PT: } \\
(\mathrm{IG}<28): 6,2 \\
(\mathrm{DP} \pm 1,4) \\
\text { PT: } \\
(\text { IG28-31): 6,5 } \\
\text { (DP } \pm 1,0) \\
\text { PT: } \\
(\text { IG32-33): } 7.4 \\
\text { (DP } \pm 1,1) \\
\text { AT: } 5,4 \\
\text { (DP } \pm 0,8)\end{array}$ & $\begin{array}{c}\text { PT: } \\
(\mathrm{IG}<28): 2.2 \\
(\mathrm{DP} \pm 0,5) \\
\text { PT: } \\
(\mathrm{IG} 28-31): 2,1 \\
(\mathrm{DP} \pm 0,3) \\
\text { PT: } \\
(\mathrm{IG} 32-33): 2,0 \\
(\mathbf{D P} \pm \mathbf{0 , 3}) \\
\text { AT: } 1,9 \\
(\mathrm{DP} \pm 0,3)\end{array}$ & \\
\hline \multirow[b]{2}{*}{$\begin{array}{l}\text { Charpak e Ruiz, 2007[15]. } \\
\text { Colômbia. }\end{array}$} & \multirow[b]{2}{*}{$\begin{array}{l}\text { Descrever a } \\
\text { evolução e a } \\
\text { composição do } \\
\text { LMPT de acordo } \\
\text { com a IG. }\end{array}$} & \multirow[b]{2}{*}{$\begin{array}{c}\text { Coleta: } 7 \text { o dia } \\
\mathbf{n}=113 \\
\text { (IG 32-36 SMN) } \\
\text { Amostra de LM de } 113 \\
\text { lactantes com IG entre } \\
32 \text { e } 36 \text { semanas. }\end{array}$} & $\mathrm{mg} \%$ & $\mathrm{~g} \%$ & \multicolumn{2}{|c|}{$\mathrm{mg} \%$} & \multirow[b]{2}{*}{$\begin{array}{l}\text { A composição do leite humano prematuro não mostrou } \\
\text { diferença nos teores de PTN, Ca ou P entre o leite anterior } \\
\text { e o leite posterior em cada período de amostragem. } \\
\text { O conteúdo de Ca e P permaneceu estável, com relação } \\
\text { Ca/P de 2:1. } \\
\text { As concentrações de PTN foram maiores nas amostras } \\
\text { iniciais e diminuíram constantemente com o passar do } \\
\text { tempo. } \\
\text { As concentrações LIP variaram muito, mas foram } \\
\text { uniformemente maiores no leite posterior em comparação } \\
\text { ao leite anterior. }\end{array}$} \\
\hline & & & $\begin{array}{c}\text { PT: } \\
\text { (IG 32 a } 35 \text { SMN) } \\
2,3 \text { a } 2 \\
\\
\text { PT: } \\
\text { (IG 36 SMN) } \\
1,9\end{array}$ & $\begin{array}{l}\text { PT: } \\
\text { Coleta do início } \\
\text { da mamada } \\
4,4 \\
\text { PT: } \\
\text { Coleta do final } \\
\text { da mamada } \\
3,4\end{array}$ & $\begin{array}{c}\text { PT: } \\
\left(1^{\circ} \mathrm{SMN}\right): \\
30\end{array}$ & $\begin{array}{c}\text { PT: } \\
\left(1^{\circ} \mathrm{SMN}\right) \\
15\end{array}$ & \\
\hline
\end{tabular}




\begin{tabular}{|c|c|c|c|c|c|c|c|}
\hline \multirow[b]{2}{*}{$\begin{array}{l}\text { Yamawaki et al., 2005[31]. } \\
\text { Japão. }\end{array}$} & \multirow[b]{2}{*}{$\begin{array}{l}\text { Determinar e } \\
\text { comparar a } \\
\text { concentração de } \\
\text { macronutrientes e a } \\
\text { composição dos } \\
\text { minerais no LM. }\end{array}$} & \multirow[b]{2}{*}{$\begin{array}{c}\text { Coleta: } 5^{\circ} \text { e } 10^{\circ} \text { dia. } \\
n=1190 \\
\text { (IG não mencionado) }\end{array}$} & \multicolumn{2}{|c|}{$\mathrm{g} / 100 \mathrm{ml}$} & \multicolumn{2}{|c|}{$\mathrm{mg} ; 100 \mathrm{ml}$} & \multirow[b]{2}{*}{$\begin{array}{l}\text { O conteúdo de LIP mostrou um aumento dependente do } \\
\text { tempo de lactação, sendo maior a partir do } 11 \text { o dia. } \\
\text { A concentração de nitrogênio da PTN diminuiu } \\
\text { significativamente durante o ano de lactação em diferentes } \\
\text { taxas, dependendo do estágio da lactação. } \\
\text { A composição de aminoácidos livres do leite humano } \\
\text { variava de acordo com o estágio da lactação, e o padrão } \\
\text { dessa variação diferia dependendo do aminoácido. }\end{array}$} \\
\hline & & & $\begin{array}{c}\mathbf{5} \mathbf{0} \mathbf{D}: \\
\text { Nitrogênio total } \\
0,29 \\
(\mathrm{DP} \pm 0,06) \\
\\
\text { Nitrog. }(\mathrm{x} 6,38) \\
1,84 \\
(\mathrm{DP} \pm 0,38) \\
\\
\mathbf{1 0} \mathbf{0} \mathbf{D}: \\
\text { Nitrogênio total } \\
0,30 \\
\text { (DP } \pm 0,07) \\
\\
\text { Nitrog. (x } 6,38) \\
2,77 \\
\text { (DP } \pm 1,14)\end{array}$ & $\begin{array}{c}5 \mathbf{0} \mathbf{D}: \\
2,68 \\
(\mathrm{DP} \pm 1,00) \\
\\
\mathbf{1 0} \mathbf{0} \mathbf{D}: \\
2,77 \\
(\mathrm{DP} \pm 1,14)\end{array}$ & $\begin{array}{c}\mathbf{5} \mathbf{0} \mathbf{D}: \\
29,3 \\
(\mathrm{DP} \pm 7,2) \\
\\
\mathbf{1 0} \mathbf{0} \mathbf{D}: \\
31,0 \\
(\mathrm{DP} \pm 9,7)\end{array}$ & $\begin{array}{c}5 \mathbf{0} \mathbf{D}: \\
15,9 \\
(\mathrm{DP} \pm 4,0) \\
\\
\mathbf{1 0} \mathbf{o} \mathbf{D}: \\
19,0 \\
(\mathrm{DP} \pm 6,1)\end{array}$ & \\
\hline \multirow[b]{2}{*}{$\begin{array}{c}\text { Bortolozo et al., 2004[13]. } \\
\text { Brasil. }\end{array}$} & \multirow[b]{2}{*}{$\begin{array}{l}\text { Analisar a } \\
\text { composição de } \\
\text { macro e } \\
\text { micronutrientes no } \\
\text { leite humano } \\
\text { coletado em } 2 \\
\text { bancos de leite. }\end{array}$} & \multirow[b]{2}{*}{$\begin{array}{c}\text { Coleta: } \\
\mathbf{n}=26 \\
\text { (leite maduro) } \\
\mathbf{n}=10 \\
\text { (colostro) } \\
\mathbf{n}=10 \\
(\mathrm{IG} \leq 36 \mathrm{SMN})\end{array}$} & \multicolumn{2}{|c|}{$\frac{1}{\mathrm{~g} / 100 \mathrm{ml}}$} & \multicolumn{2}{|c|}{$\mathrm{mg} / 100 \mathrm{ml}$} & \multirow[b]{2}{*}{$\begin{array}{l}\text { A composição variou tanto entre os tipos de leite quanto } \\
\text { entre doadoras do mesmo leite. } \\
\text { Os valores de micro e macronutrientes foram inferiores às } \\
\text { necessidades dos recém-nascidos de baixo peso. } \\
\text { Ocorreu variação significativa na PTN para o leite AT em } \\
\text { relação ao colostro e ao LMPT ( } p<0,005) \text {. } \\
\text { Para LIP as concentrações não apresentaram variação } \\
\text { significativa }(p>0,005) \text {. }\end{array}$} \\
\hline & & & $\begin{array}{l}\text { CL: } 1,71 \\
(\mathrm{DP} \pm 0,29) \\
\text { PT: } 1,72 \\
(\mathrm{DP} \pm 0,4) \\
\text { AT: } 1,07 \\
(\mathrm{DP} \pm 0,22)\end{array}$ & $\begin{array}{c}\text { CL: } 2,48 \\
(\mathrm{DP} \pm 0,91) \\
\text { PT: } 2,48 \\
(\mathrm{DP} \pm 0,76) \\
\text { AT: } 2,56 \\
(\mathrm{DP} \pm 0,8)\end{array}$ & $\begin{array}{c}\text { CL: } 22,75 \\
(\mathrm{DP} \pm 10,24) \\
\text { PT: } 22,03 \\
(\mathrm{DP} \pm 9,39) \\
\\
\text { AT: } 17,88 \\
(\mathrm{DP} \pm 5,56)\end{array}$ & $\begin{array}{l}\text { CL: } 9,31 \\
(\mathrm{DP} \pm 4,3) \\
\text { PT: } 8,47 \\
(\mathrm{DP} \pm 2,43) \\
\text { AT: } 9,98 \\
(\mathrm{DP} \pm 1,72)\end{array}$ & \\
\hline \multirow[b]{2}{*}{$\begin{array}{l}\text { Pishva e Hemmati, 2004[32]. } \\
\text { Irã. }\end{array}$} & \multirow[b]{2}{*}{$\begin{array}{c}\text { Determinar os teores } \\
\text { de PTN, LIP e Ca } \\
\text { no Leite de lactantes } \\
\text { de PT e AT. }\end{array}$} & \multirow[b]{2}{*}{$\begin{array}{c}\text { Coleta: } 20 \text { a } 50 \text { dia. } \\
\mathbf{n}=20 \\
(\mathrm{IG} \leq 37 \mathrm{SMN}) \\
\mathbf{n}=20 \\
(\mathrm{IG} 38-40 \mathrm{SMN})\end{array}$} & \multicolumn{2}{|c|}{$\frac{1}{\mathrm{mg} / \mathrm{dl}}$} & \multicolumn{2}{|c|}{$\mathrm{mg} / \mathrm{dl}$} & \\
\hline & & & $\begin{array}{l}\text { PT: } 11.95 \\
(\mathrm{DP} \pm 4.34) \\
\text { AT: } 10.88 \\
(\mathrm{DP} \pm 2.11)\end{array}$ & $\begin{array}{l}\text { (colesterol) } \\
\text { PT: } 127.9 \\
\text { (DP } \pm 84.80) \\
\\
\text { (colesterol) } \\
\text { AT: } 186.10 \\
\text { (DP } \pm 122.1)\end{array}$ & $\begin{array}{c}\text { PT: } 22.18 \\
(\mathrm{DP} \pm 5.10) \\
\\
\text { AT: } 20.76 \\
(\mathrm{DP} \pm 5.56)\end{array}$ & - & $\begin{array}{l}\text { Não foram encontradas diferenças significativas na } \\
\text { concentração de colesterol entre o LMPT e LMAT ( } p \\
0,070) \text {. Contudo, a concentração de colesterol aumentou } \\
\text { durante a lactação progressiva em bebês PT ( } p<0,001) \text {. } \\
\text { A concentração de Ca foi relativamente similar em LMPT e } \\
\text { LMAT }(p 0,368) \text {. }\end{array}$ \\
\hline
\end{tabular}




\begin{tabular}{|c|c|c|c|c|c|c|c|}
\hline \multirow[b]{2}{*}{$\begin{array}{l}\text { Butte et al., 1983[28]. } \\
\text { USA. }\end{array}$} & \multirow[b]{2}{*}{$\begin{array}{l}\text { Determinar as } \\
\text { concentrações de } \\
\text { nitrogênio proteico, } \\
\text { nitrogênio não- } \\
\text { proteico, LIP, Ca e } \\
\text { P. }\end{array}$} & \multirow[b]{2}{*}{$\begin{array}{c}\text { Coleta: } 14^{\circ} \text { dia. } \\
\mathbf{n}=13 \\
(\mathrm{IG} \geq 37 \mathrm{SMN}) \\
\mathbf{n}=8 \\
(<37 \mathrm{SMN})\end{array}$} & $\mathrm{mg} / \mathrm{dl}$ & $\mathrm{g} / \mathrm{dl}$ & \multicolumn{2}{|c|}{$\mathrm{mg} / \mathrm{l}$} & \multirow[b]{2}{*}{$\begin{array}{l}\text { A concentração de LIP não se alterou ao longo do tempo e } \\
\text { não houve diferenças significativas entre os grupos. } \\
\text { O teor de Ca do leite não se alterou. } \\
\text { A concentração média de Ca do LMAT foi } \\
\text { significativamente maior que do LMPT ( } p=0,05) \text {. O nível } \\
\text { médio de P de LM AT foi significativamente maior que o de } \\
\text { LMPT ( } p<0,03) \text {. } \\
\text { Maiores concentrações de nitrogênio proteico no LMPT } \\
\text { foram expressos no 14o dia. }\end{array}$} \\
\hline & & & $\begin{array}{c}\text { Nitr. prot } \\
\text { PT: } 241,0 \\
\text { (DP } \pm 18) \\
\text { AT: } 207,0 \\
\text { (DP } \pm 29 \text { ) } \\
\text { Nitr. não prot. } \\
\text { PT: } 57,0 \\
\text { (DP } \pm 16 \text { ) } \\
\text { AT: } 55,0 \\
\text { (DP } \pm 12 \text { ) }\end{array}$ & $\begin{array}{l}\text { PT: } 3,45 \\
(\mathrm{DP} \pm 0.94) \\
\text { AT: } 3,79 \\
(\mathrm{DP} \pm 1.00)\end{array}$ & $\begin{array}{l}\text { PT: } 214,0 \\
(\mathrm{DP} \pm 48) \\
\text { AT: } 255,0 \\
(\mathrm{DP} \pm 53)\end{array}$ & $\begin{array}{l}\text { PT: } 149,0 \\
(\mathrm{DP} \pm 38) \\
\text { AT: } 179,0 \\
(\mathrm{DP} \pm 36)\end{array}$ & \\
\hline \multirow[b]{2}{*}{$\begin{array}{c}\text { Lemons } e t \text { al., } 1982^{[12]} . \\
\text { USA. }\end{array}$} & \multirow[b]{2}{*}{$\begin{array}{l}\text { Avaliar a } \\
\text { composição do } \\
\text { LMPT e de LMAT } \\
\text { no mesmo período } \\
\text { de lactação. }\end{array}$} & \multirow[b]{2}{*}{$\begin{array}{c}\text { Coleta: } 7 \text { o dia. } \\
\mathbf{n}=20 \\
\text { (IG } 27-37 \text { SMN). } \\
\mathbf{n}=7 \\
\text { (IG } 39-41 \text { SMN). }\end{array}$} & \multicolumn{2}{|c|}{$\mathrm{g} / \mathrm{L}$} & \multicolumn{2}{|c|}{$\mathrm{mg} / \mathrm{L}$} & \multirow[b]{2}{*}{$\begin{array}{l}\text { O LMPT tem maiores concentrações de nitrogênio total e } \\
\text { nitrogênio proteico que o leite LMAT. } \\
\text { Não houve diferenças de LMAT para LMPT em relação ao } \\
\text { Ca. } \\
\text { Nenhuma diferença significativa foi encontrada entre os } \\
\text { grupos LMPT e LMAT em relação à gordura. }\end{array}$} \\
\hline & & & $\begin{array}{c}\text { Nitr. prot } \\
\text { PT: } 2,76 \\
\text { (DP } \pm 0,18) \\
\text { AT: } 2,55 \\
\text { (DP } \pm 0,13) \\
\\
\text { Nitr. não prot. } \\
\text { PT: } 0,31 \\
\text { (DP } \pm 0,03) \\
\text { AT: } 0.32 \\
\text { (DP } \pm 0,03)\end{array}$ & $\begin{array}{l}\text { PT: } 3,10 \\
(\mathrm{DP} \pm 0,17) \\
\mathbf{A T}: 2.98 \\
(\mathrm{DP} \pm 0,28)\end{array}$ & $\begin{array}{l}\text { PT: } 293,11 \\
(\mathrm{DP} \pm 16,4) \\
\\
\text { AT: } 293.14 \\
(\mathrm{DP} \pm 7,61)\end{array}$ & $\begin{array}{l}\text { PT: } 134,48 \\
(\mathrm{DP} \pm 6,45) \\
\text { AT: } 169.30 \\
(\mathrm{DP} \pm 8,55)\end{array}$ & \\
\hline \multirow{2}{*}{$\begin{array}{l}\text { Gross et al., } 1980^{[11]} . \\
\text { USA. }\end{array}$} & \multirow[b]{2}{*}{$\begin{array}{l}\text { Determinar a } \\
\text { composição } \\
\text { nutricional do leite } \\
\text { no primeiro mês } \\
\text { pós-parto. }\end{array}$} & \multirow[b]{2}{*}{$\begin{array}{c}\text { Coleta: } 70 \text { dia. } \\
\mathbf{n}=18 \\
\text { (IG:38-42 SMN). } \\
\mathbf{n}=33 \text { (IG:28-36). }\end{array}$} & \multicolumn{2}{|c|}{$\mathrm{g} / \mathrm{dl}$} & \multicolumn{2}{|c|}{$\mathrm{mg} / \mathrm{l}$} & \multirow[b]{2}{*}{$\begin{array}{l}\text { As concentrações de proteína do LMPT foram } \\
\text { significativamente maiores. } \\
\text { As concentrações de Ca e P do leite produzido pelos dois } \\
\text { grupos foram semelhantes. } \\
\text { As concentrações de LIP foram menores no leite obtido no } \\
3^{2} \text { dia, aumentaram no 7o dia e permaneceram constantes. }\end{array}$} \\
\hline & & & $\begin{array}{l}\text { PT: } 2,44 \\
(\mathrm{DP} \pm 0,15) \\
\text { AT: } 1,87 \\
(\mathrm{DP} \pm 0,08)\end{array}$ & $\begin{array}{c}\text { PT: }: 3,81 \\
(\mathrm{DP} \pm 0,21) \\
\text { AT: } 3,06 \\
(\mathrm{DP} \pm 0,46)\end{array}$ & $\begin{array}{l}\text { PT: } 247 \\
(\mathrm{DP} \pm 16) \\
\text { AT: } 254 \text { (DP } \\
\quad \pm 11)\end{array}$ & $\begin{array}{l}\text { PT: } 142 \\
(\mathrm{DP} \pm 10) \\
\text { AT: } 151 \\
(\mathrm{DP} \pm 18)\end{array}$ & \\
\hline \multirow[b]{2}{*}{$\begin{array}{c}\text { Sann et al, 1980[30]. } \\
\text { França. }\end{array}$} & \multirow[b]{2}{*}{$\begin{array}{c}\text { Analisar as } \\
\text { concentrações do } \\
\text { LMPT e do LMAT. }\end{array}$} & \multirow[b]{2}{*}{$\begin{array}{c}\text { Coleta: } 70 \text { ao } 140 \text { dia. } \\
\mathbf{n}=61 \\
\begin{array}{c}\text { (IG: } 38-41 \mathrm{SMN}) \\
\mathbf{n}=41 \\
\text { (IG: } 26-35 \mathrm{SMN})\end{array}\end{array}$} & \multicolumn{2}{|c|}{$\mathrm{g} / 100 \mathrm{Kcal}$} & \multicolumn{2}{|c|}{$\mathrm{mmol} / \mathrm{kcal}$} & \multirow[b]{2}{*}{$\begin{array}{l}\text { Não houve diferença na composição de PT e AT em } \\
\text { qualquer estáaio da lactação. } \\
\text { A alimentação precoce de PT com LM colostral e } \\
\text { transicional pode ser mais apropriada, contudo, os } \\
\text { requerimentos de Ca não foram atingidos. }\end{array}$} \\
\hline & & & $\begin{array}{l}\text { PT: } 18 \\
(\mathrm{DP} \pm 6) \\
\text { AT: } 15,7 \\
(\mathrm{DP} \pm 3)\end{array}$ & $\begin{array}{c}\text { PT: } 25 \\
(\mathrm{DP} \pm 8) \\
\text { AT: } 27 \\
(\mathrm{DP} \pm 14)\end{array}$ & $\begin{array}{c}\text { PT: } 7,62 \text { (DP } \\
\quad \pm 2,15) \\
\text { AT: } 7.09 \\
(\mathrm{DP} \pm 1,2)\end{array}$ & $\begin{array}{c}\text { PT: } 4,9 \\
(\mathrm{DP} \pm 1,4) \\
\text { AT: } 3,36 \\
(\mathrm{DP} \pm 1,16)\end{array}$ & \\
\hline
\end{tabular}

Legenda: $\mathrm{AT}=$ a termo; $\mathrm{PT}=$ pré-termo $\mathrm{CL}=$ colostro; $\mathrm{IG}=$ idade gestacional; $\mathrm{LM}=$ leite materno; $5 \circ \mathrm{D}=$ quinto dia; $10 \circ \mathrm{D}=$ décimo dia; $\mathrm{SMN}=$ semana(s); Nitr. Prot= nitrogênio proteico; Nitr. não prot.= Nitrogênio não proteico. 
Os estudos realizados por Lemons et al..$^{[12]} \mathrm{e}$ Bortoloso et al.[13] também relatam concentrações significativamente mais altas de nitrogênio total e nitrogênio proteico no LMPT durante as primeiras 4 semanas de lactação ( $>$ <0,005). Análises multivariadas realizadas por Hsu et al. ${ }^{[14]}$ verificaram uma relação inversa entre o conteúdo de PTN e o peso ao nascer, indicando que os componentes do LM foram provavelmente ajustados de acordo com as necessidades das crianças. Os autores mencionaram que as concentrações de nutrientes foram significativamente influenciadas pela idade gestacional. Além disso, o LMPT com baixo peso ao nascer continham mais PTN. Charpak e Ruiz[15] encontraram dados parecidos em amostras de leite retiradas entre o 1 e o 8o dia após o parto, sugerindo variação inversamente da proteína em relação a idade pós-conceitual e a idade pós-natal.

Em relação ao conteúdo de aminoácidos, Chuang et al.[16] verificaram no colostro de LMPT valores elevados de prolina $(19,3 \%)$, alanina $(7,8 \%)$, valina $(7,4 \%)$, ácido glutâmico $(7,3 \%)$ e taurina $(5,1 \%)$. Com exceção da fosfoetanolamina, hidroxiprolina, asparagina e ácido a-amino-hbutírico, as amostras de LMPT continham quatro vezes mais aminoácidos livres do que o LMAT. Ademais, as PTN totais no colostro foram significativamente maiores em ambos os grupos, quando comparado ao leite de transição.

Uma tendência de diminuição gradativa na concentração das PTN do soro a partir do início da lactação foi observada por Kunz e Lönnerdal[17] resultando em uma relação proteína/caseína de 9:1 no início da lactação, 6:4 no leite maduro e 5:5 no final da lactação. As diferenças marcantes na concentração de caseína ocorrem entre o 4o dia de lactação, variando entre $1,2 \mathrm{mg} / \mathrm{ml} \mathrm{a} 4,6 \mathrm{mg} / \mathrm{ml}$. Armaforce et al. ${ }^{18]}$ ao realizarem comparações proteômicas entre o LMPT e LMAT, evidenciaram uma redução dos níveis de $\beta$-caseína e $\alpha$-caseína, além do aparecimento de produtos adicionais no LMPT, correspondendo a produtos de hidrólise de baixo peso molecular das caseínas. Molinari et al.[19] através de análises de glicosilação de PTN e a fosforilação de B-caseína em leite de puérperas de recém-nascidos AT e PT, verificaram uma abundante diminuição na concentração das PTN do LMAT e LMPT durante a lactação $(p \leq 0,05)$. Os níveis de fosforilação no LMPT aumentaram ao longo da lactação e a ß-caseína estava presente em 5 isoformas de caseína fosforilada de intensidade relativamente uniforme em 37,5\% das amostras, nas $62,5 \%$ amostras restantes nenhuma mudança no padrão de fosforilação foi observada ao longo do tempo. No LMPT foram constatadas maiores concentrações de nitrogênio total e de atividade de plasminas.

Molinari et al.[19] encontraram 3 enzimas digestivas diferencialmente expressas em LMAT e LMPT. Entretanto, a lipase estimulada por sais biliares (BSSL) estava presente em níveis mais alto no LMPT, enquanto a biotinidase (BTD) e a lipoproteína lipase (LPL) estavam em maiores níveis no LMAT.

Em outro estudo, Molinari et al20] identificaram 415 PTN distintas no LMPT e LMAT, entre o 7o e 28o dias após o parto. Destas, 100 PTN foram identificadas apenas entre o 15ㅇ ao 28 o dias. Houve uma equivalência de 174 PTN em ambos os grupos. No LMPT 5 PTN tinham valores significativamente maiores (albumina sérica, lipase ativada por sal biliar, desulfurase de cisteína, lactotransferrina e lisozima-C) que em LMAT e 10 PTN (receptor de imunoglobulina polimérica, clusterin, proteína induzível por prolactina, lactadherin, alfa-lactalbumina, mucina-4, Ig HG3, biotinidase, gama-glutamil transpeptidase e complemento C3) estavam presentes em um nível significativamente maior no LMAT. Os autores relatam ainda que, das 415 PTN, 24\% estavam envolvidas na resposta imune e $28 \%$ eram direcionadas ao metabolismo celular e crescimento celular.

\section{Lipídeos}

No estudo de Bitman et al.[21] as concentrações de ácidos graxos de cadeia média aumentaram do colostro para o leite maduro e foram maiores no LMPT. Os ácidos graxos poli-insaturados de cadeia longa também foram os mais elevados no colostro, além de estarem em maior quantidade no LMPT. Em relação aos ácidos graxos de cadeia curta, seu conteúdo estava aproximadamente o dobro no LMPT, quando comparado ao LMAT. Para os autores, os bebês prematuros que ingerem o leite da própria progenitora recebem $17 \%$ do lipídio total como ácido graxo de cadeia média enquanto os bebês AT recebem em torno de $10 \%$. 
No estudo realizado por Berenhauser ${ }^{[22]}$ foram observados, tanto no grupo LMPT como no grupo LMAT, diferenças significativas para a maior parte dos ácidos graxos saturados, monoinsaturados e poli-insaturados. Entre essas diferenças, o autor relata nos grupos LMPT e LMAT uma redução significativa dos níveis de ácido docosahexaenóico (DHA) do colostro para o leite maduro. O ácido elaídico estava significativamente mais elevado no LMAT maduro e significativamente mais elevado no colostro do LMPT. O ácido rumênico elevou-se significativamente no LMPT maduro e foi significativamente menor no colostro do grupo LMPT, quando comparado ao colostro do grupo LMAT. O ácido linoléico apresentou valores significativamente mais elevados no LMPT, tanto no colostro como no maduro, quando comparado com colostro e LMAT maduro.

Fumeaux et al.[23] anallisando composição de macronutrientes no leite humano constataram um sutil aumento do LIP no início da lactação, tanto no LMAT quanto no LMPT. Porém, o conteúdo LIP parece permanecer constante após esse período inicial. O LMPT continha significativamente mais LIP e seguiam uma tendência de ser mais calórico nas duas primeiras semanas de lactação, enquanto o LMAT revelou maior LIP e maior conteúdo de energia entre a 3a e 8a semana de lactação. Os autores encontraram uma possível associação entre os neonatos do sexo masculino e o conteúdo de LIP do leite de suas progenitoras, sendo essa associação de gênero mais forte no LMPT. Ao comparar o teor de gordura do LMAT ao LMPT, de acordo com a idade pós-menstrual, duas diferenças significativas foram observadas: para a idade gestacional de 42 semanas o LMPT apresentou maior teor de LIP, em contrapartida, para a idade gestacional de 47 semanas o teor de LIP do LMPT foi menor.

Ao realizar uma análise de dados agrupados, que contou com 4374 amostras de LMAT e 1017 de LMPT, Floris et al.[24] relataram uma estabilidade dos ácido palmítico, linoleico e oleico ao longo do tempo, possivelmente devido a sua fonte primária ser proveniente do tecido adiposo materno. Já os poliinsaturados de cadeia longa, como o ácido araquidônico (ARA) e DHA, aparentemente diminuiu conforme o tempo de amamentação e os ácidos graxos de cadeia média aumentaram, com o passar dos dias. Uma comparação da variação inter estudos demonstrou consistência notável nos resultados envolvendo os ARA. Os autores levantaram a hipótese do ácido palmítico ter mais probabilidade de ser geneticamente regulado, em comparação ao ácido oleico e o ácido esteárico, assim como uma origem mais dietética dos níveis de ARA no leite materno, quando comparado ao DHA. Em conclusão, os ácidos graxos flutuam de acordo com padrões distintos ao longo da lactação, onde muitos desses padrões são comparáveis entre LMAT e LMPT. No entanto, as análises este estudo demonstram claramente a diversidade nas espécies de ácidos graxos e a falta do conhecimento aos efeitos fisiológicos individuais ou sinérgicos desta heterogeneidade nos lactentes.

\section{$\mathrm{Ca}$}

Ao descrever a evolução e a composição do LMPT de acordo com a idade gestacional e a idade pós-natal Charpak e Ruiz ${ }^{[15]}$ apontaram uma permanência estável de $\mathrm{Ca}$ e $\mathrm{P}$ durante todos os períodos observados, com uma relação de Ca para $\mathrm{P}$ de 2:1. Trabalhos anteriores descreveram uma constância na concentração de Ca durante todo o primeiro mês de lactação, tanto para o LMPT quanto para LMAT ${ }^{[11,12] .}$

Conforme referido por Hsu et al..14] não houve correlação entre o número de semanas pósparto e os níveis de Ca, contudo foi observado que quanto maior o peso corporal ao nascer e/ou a idade gestacional maior os níveis de Ca no leite. Os níveis de fosfato foram menores para o LMPT, sendo observado aumento do fosfato conforme as semanas pós-parto nos dois grupos estudados. Lemons et al. [12] também encontraram teores significativamente menores de $\mathrm{P}(\phi \leq 0,05)$ no LMPT.

Atkinson et al.[25] demonstraram em seu estudo uma diminuição significativa do Ca conforme a progressão da lactação $(p<0,05)$, tanto no LMAT quanto no LMP'T. No LMAT o Ca diminuiu após o 7으 dia, permanecendo estável no período subsequente. No LMPT a concentração de Ca diminuiu entre o 13o e o 17o dia pós-parto. Com relação ao $\mathrm{P}$ ocorreu um aumento da concentração dos níveis iniciais $(p<0,01)$ entre o 7 o e o 10 o dia de lactação em mães AT. Os autores relataram uma tendência semelhante entre os grupos de valores 
maiores de Ca para valores menores de $\mathrm{P}$, sendo essa relação $\mathrm{Ca} / \mathrm{P}$ significativamente maior do 3 o ao 5 o dia de lactação.

Em estudo de Sabatier et al.[26], os autores observaram que o conteúdo mineral do LMAT e LMPT e as alterações paralelas ao longo do período de lactação, constatou que o $\mathrm{Ca}$ foi o segundo elemento mais concentrado em ambos os leites maternos, sendo menor apenas do potássio. Não foi encontrada diferença estatística nas concentrações de $\mathrm{P}$ e de Ca entre o LMPT e LMAT na mesma idade pós-parto. As razões de massa de Ca para $\mathrm{P}$ variaram entre 1,57 e 2,32 no LMPT e de 1,84 e 2,22 no LMAT, com uma diferença estatisticamente significante entre os grupos encontrados na $1 \underline{a}$ semana $(p=0,042)$ e na $2 \underline{a}$ semana $(p=0,018)$. Além disso, as concentrações de $\mathrm{P}$ no leite aumentaram durante a primeira semana pós-parto e depois diminuíram durante a lactação e as concentrações de $\mathrm{Ca}$ foram mais altas na $1^{\mathrm{a}}$ semana e depois diminuíram gradualmente durante a lactação.

Codo et al..$^{[27]}$ relataram em sua pesquisa que o Ca não mostrou diferença estatística significante entre o LMPT e o LMAT em relação às suas concentrações, durante o mesma idade pós-parto. Entretanto, o conteúdo de $\mathrm{P}$ foi significativa maior no LMPT na 1a semana pós-parto. Os autores ressaltam que tanto $\mathrm{P}$ quanto Ca só atingiriam os níveis diários de ingestão recomendados pelas Sociedades Europeias se fossem ofertados ao bebê pelo menos $60 \mathrm{~mL}$ de leite a cada 3 horas. Visto isso, como o volume de leite consumido por um recémnascido PT é limitado devido às condições clínicas, o conteúdo de Ca não seria suficiente para garantir as demandas nutricionais desse mineral.

\section{DISCUSSÃO}

A partir do exposto verifica-se que componentes do LM como PTN e LIP apresentam ajustes em função do tipo de gestação apresentada pelo recém nascido. Como exemplo, a tendência decrescente observada da PTN à medida que aumenta a idade gestacional e da relação inversa entre o conteúdo de PTN do LM humano e o peso ao nascer do bebê [13,15], além das diferentes expressões encontradas entre os aminoácidos e a maior quantidade de PTN presentes no LMPT ${ }^{[24,14,19]}$.
O LMPT possui concentrações maiores de PTN nos primeiros dias de vida, sugerindo que prematuros alimentados com o leite da própria mãe receberiam $67 \%$ a mais de PTN, em comparação ao mesmo volume de leite proveniente de doadores ${ }^{[11,12]}$. Segundo Butte et al.[28] há evidências de um equilíbrio hormonal regulando o metabolismo associados aos períodos gestacionais mais curtos, o que poderia alterar a síntese de PTN. Contudo, não se sabe ao certo o real mecanismo para os altos níveis proteicos no LMPT.

A tendência da diminuição gradativa na concentração da caseína observada ao decorrer da gestação sinalizam diferentes mecanismos na regulação de sua síntese e secreção ${ }^{[14]}$. Essa regulação da caseína, além da constatação de maiores concentrações de nitrogênio total e da atividade de plasminas nas amostras de LMPT analisadas, sugerem um mecanismo fisiológico que ajustaria a expressão de enzimas e/ou PTN para modificar a digestibilidade proteica nos lactentes ${ }^{18]}$.

Conforme descrito por Hsu et al. [17] os LIP no leite humano aumentaram nos grupos estudados de forma gradativa durante as semanas de lactação pós-parto, enquanto os níveis de PTN diminuíram lentamente. Charpak e Ruiz ${ }^{[15]}$ também observaram diferenças nas concentrações de LIP em períodos diferentes da mesma mamada. Consistentemente, o leite tem um teor de LIP elevado no início do aleitamento em relação ao leite do final do aleitamento. Os autores ressaltaram que a utilização do leite retirado no início da mamada pode otimizar as necessidades nutricionais de bebês PT devido ao aumento na oferta da densidade calórica, o que seria uma alternativa simples, segura, facilmente acessível e com custos mínimos.

A maior concentração dos ácidos graxos de cadeia média no leite de prematuros pode melhorar a absorção deste nutriente, devido a um processo de hidrólise mais efetivo. Esse fato torna a composição do LMPT mais adequada ao sistema digestivo do prematuro, do qual sofre pelos baixos níveis de lipase pancreática e sais biliares ${ }^{[21]}$. Além disso, as diferentes expressões enzimáticas no leite humano encontradas por Molinari et al.[19] podem suprir a falta de enzimas endógenas no suco pancreático imaturo dos recémnascidos, permitindo a digestão eficiente de 
triacilgliceróis e biotinas, de relevante importância para o crescimento e desenvolvimento infantil. Contudo, se os níveis mais altos de BSSL no LMPT podem promover crescimento adicional em bebês prematuros, os níveis mais baixos de BTD e LPL podem ser prejudiciais, tornando os padrões de expressão encontrados nesse estudo desafiadores a uma explicação teleológica.

Vale ressaltar a importância do estudo realizado por Berenhauser ${ }^{[22]}$, no qual foi demonstrada uma concentração significativamente maior de ácido linoleico no colostro do LMPT. Esse nutriente tem papel fundamental no crescimento e desenvolvimento do sistema nervoso central, de precursor de ácidos graxos de cadeia longa como os ARA, eicosadienóico e eicosatrienóico.

No que diz respeito aos parâmetros de $\mathrm{Ca}$ e $\mathrm{P}$, a relação 2:1 permaneceu estável na maioria dos estudos. Todavia, Hsu et al.[14] e Lemons et al.[12] encontraram níveis menores de $\mathrm{P}$ no leite de recémnascidos e Atkinson et al ${ }^{[26]}$ relatam a tendência do $\mathrm{P}$ menor e do Ca maior no início da lactação. Hsu et al. ${ }^{[4]}$ ainda mencionam a influência do peso e da idade gestacional no nível de Ca no leite.

Underwood ${ }^{[29]}$ encontrou conteúdo de Ca significativamente menor no leite de mães que deram a luz a prematuros extremos e esse conteúdo determinado não parece aumentar com o tempo. $\mathrm{O}$ autor ressalta que os valores de Ca encontrados em estudo de LMPT com baixo peso extremo pode não refletir os mesmos resultados de análises realizados em leite de mães de bebês prematuros maiores. Da mesma maneira, não poderia haver contraposto ao leite humano pasteurizado, já que o mesmo não fornece os nutrientes ou as moléculas bioativas equivalentes ao próprio LM não pasteurizado.

Em um estudo realizado por Codo et al.[27], o processo de pasteurização e evaporação do LMAT levou a uma redução significativa no conteúdo de Ca $(p=0,003)$ e do P $(p=0,004)$, deixando esses minerais abaixo dos valores recomendados. Esta condição agravaria mais o aporte destes minerais para os bebês, principalmente os prematuros. Além disso, mesmo considerando a quantidade de $\mathrm{Ca}$ e $\mathrm{P}$ do LMPT coletada a beira leito, a oferta diária recomendada só seria alcançada com o consumo de um grande volume de leite materno. Considerando o valor médio de Ca determinado no leite coletado (197,3 mg/L), um recém-nascido teria que consumir mais de $500 \mathrm{ml}$ de LM por dia para atingir o valor recomendado. Esses achados vão de encontro aos dados descritos por Sann et al. ${ }^{30]}$ a partir dos quais foi demonstrado que a alimentação precoce de PT com LMPT, seja ele colostro ou de transição, apesar de ser mais apropriado nutricionalmente ao recém-nascido, não atinge o requerimento de $\mathrm{Ca}$ necessário para o pleno desenvolvimento.

Diversos métodos de coleta do leite humano foram descritos entre os artigos estudados, havendo diferenças quanto aos horários e seus procedimentos. Entre estes, podemos mencionar o utilizado por Yamawaki et al.[31], com coleta de aproximadamente $50 \mathrm{ml}$ de leite humano, em um tempo intermediário durante a sucção; o estudo de Pishva e Hemmati[32], no qual se utilizou o leite coletado entre as 10:00 e 11:00 horas da manhã, pelo esvaziamento manual ou mecânico da mama; e o estudo de Bauer e Gerss ${ }^{[3]}$, com coletas de amostras realizadas pelas mães em suas casas, retiradas uma vez por semana durante um período de 24 horas (das 8:00 às 8:00) para controlar possíveis variações diurnas. Os diferentes tipos de abordagens descritos nestes trabalhos acabam dificultando uma avaliação comparável do conteúdo nutricional do leite materno, seja de modo quantitativo ao qualitativo. Outro ponto em questão seria em relação aos diferentes métodos de ordenha. Pessoto ${ }^{[34]}$ notou variação na média da concentração de proteína ao avaliar a lactação em mães de recémnascido PT segundo diferentes métodos de ordenha (manual, com bomba manual ou com bomba elétrica). Entretanto, na avaliação ao longo do tempo não houve variação significativa.

Por fim, apesar dos avanços em técnicas específicas de análises de elementos-traço em materiais biológicos, como o leite materno, muitos resultados apresentados nos estudos ainda são inconclusivos. Além disso, as comparações da concentração de elementos no LM entre os diversos estudos são dificultadas não somente por diferenças no âmbito analítico, mas também pelas modificações nutricionais que podem ocorrer em função de fatores relacionados aos hábitos alimentares maternos e concentrações de nutrientes nos alimentos. Assim, muitas vezes não é possível concluir se as diferenças são reais, se representam a variabilidade de padrões 
biológicos, alimentares ou ainda, se decorrem de dificuldades analíticas ${ }^{[3]}$.

\section{CONCLUSÃO}

Há um consenso entre os estudos a respeito do aumento progressivo do conteúdo de LIP no leite humano nas primeiras semanas pós-parto, e comportamento inverso acerca do conteúdo proteico, que diminui na medida em que avançam os dias de lactação. Adicionalmente, verifica-se a carência de Ca no leite materno, seja no LMPT a beira leito ou no leite pasteurizado de doadoras, o que não supri as necessidades nutricionais de lactentes PT.

De modo a responder a pergunta central que norteou esta revisão, foram observados diferenças nutricionais na comparação do LMAT com o LMPT. Como justificativa para o cenário, verificou-se a possível adaptação fisiológica e de condutas nutricionais comparando as diferentes genitoras. Outro ponto de possível diferença decorreu da diversidade de métodos analíticos utilizados ao longo dos estudos. Em suma e ponderando a partir destas colocações, há possibilidade de existência de particularidades nutricionais na composição do leite de genitoras de PT quando comparadas a genitoras de AT, possivelmente pela necessidade diferenciada dos bebês PT.

Os resultados desta revisão contribuem para o monitoramento das carências nutricionais e elaboração de estratégias individualizadas para genitoras PT e AT e para os respectivos bebês. Neste sentido, estes dados permitem a particularização para o aleitamento materno exclusivo e a compreensão das especificidades fisiológicas e nutricionais nestas diferentes condições.

\section{REFERÊNCIAS}

[1] Witkowska-Zimny M, Kamińska-El-Hassan E, Wróbel E. Milk Therapy: Unexpected Uses for Human Breast Milk. Published online. 2019;5:944.

[2] Costa LKO, Queiroz LLC, Queiroz RCCS, Ribeiro TSF, Fonseca MSS Importância do aleitamento materno exclusivo: uma revisão sistemática da literatura. Revista Ciência da Saúde. 2013; 1:39-46.

[3] WHO. Recommended definitions, terminology and format for statistical tables related to the perinatal period and use of a new certificate for cause of perinatal deaths. Modifications recommended by FIGO as amended October 14, 1976. Acta Obstet Gynecol Scand. 1977; 56:247-253.

[4] Leveno KJ, Cunningham FG, Alexander JM, Room SL, Casey BM, Dashe JS, Sheffield JS, Roberts SW. Manual de obstetrícia de Williams: complicações na gestação. 22.ed. Islabão AG, tradutor. Porto Alegre: Artmed, 2010.

[5] Fiocruz. Doação de leite humano auxilia desenvolvimento de prematuros - Agência Fiocruz notícias [Internet]. 2017 [acesso em 2019 jul 07]. Disponível em: https://agencia.fiocruz.br/doacao-deleite-humano-auxilia-desenvolvimento-de-prematuros.

[6] Moreira MEL, Rocha AD. Nutrição do recém-nascido prematuro. In: Moreira MEL, Lopes JMA, Carvalho M. Recém-nascido de alto risco: Teoria e prática do cuidador. Rio de Janeiro: Ed. Fiocruz; 2004. p. 217-242.

[7] Moura EC. Nutrição. In: Carvalho MR, Tavares LAM. Amamentação: bases científicas. 3.ed. Rio de Janeiro: Guanabara Koogan; 2014.

[8] Dominica A, Gidrewicz DA, Fenton TR. A systematic review and meta-analysis of the nutrient content of preterm and term breast milk. BMC Pediatric. 2014; 14:216.

[9] Sabatier M, Garcia-Rodenas LC, de Castro CA, Kastenmayer P, Vigo M, Dubascoux S et al. Longitudinal Changes of Mineral Concentrations in Preterm and Term Human Milk from Lactating Swiss Women. Nutrients. 2019; 11:1855.

[10] Silva CM, Pellegrinelli ALR, Pereira SCL, Passos LR, dos Santos LC. Práticas educativas segundo os "Dez passos para o sucesso do aleitamento materno" em um Banco de Leite Humano. Ciência \& Saúde Coletiva. 2017; 5:1661-1671.

[11] Gross SJ, David R, Baumam L, Tomarelli RM. Nutritional composition of milk produced by mothers delivering preterm. The Journal of Pediatrics. 1980; 4:641644.

[12] Lemon JA, Moye L, Hall D, Simmons D. Differences in the Composition of Preterm and Term Human Milk during Early Lactation. Pediatric Research. 1982; 16:113117. 
Adaptações do leite em mães de recém-nascidos prematuros. Ferreira \& Moraes

[13] Bortolozo EAFQ, Tiboni EB, Cândido LMB. Leite humano processado em bancos de leite para o recém nascido de baixo peso: análise nutricional e proposta de um novo complemento. Rev Panam Salud Publica. 2004; 3:199-205.

[14] Hsu YC, Chen CH, Lin MC, Tsai CR, Liang JT, Wang TM. Changes in Preterm Breast Milk Nutrient Content in the First Month. Journal Pediatrics and Neonatology. 2014; 55:449-454.

[15] Charpak N, Ruiz JG. Breast milk composition in a cohort of pre-term infants' mothers followed in an ambulatory programme in Colombia. Foundation Acta Pædiatrica. 2007; 96:1755-1759.

[16] Chuang CK, Lin SP, Lee HC, Wang TJ, Shih YS, Huang FY, Yeung CY. Free Amino Acids in Full-Term and Pre-Term Human Milk and Infant Formula. Journal of Pediatric Gastroenterology and Nutrition. 2005; 4:496500 .

[17] Kunz C, Lönnerdal B. Re-evaluation of the whey protein/casein ratio of human milk. Acta Paediatrica. 1992; 81(2):107-112.

[18] Armaforte E, Curran E, Huppertz T, Ryan CA, Caboni MF, O'Connor PM et al. Proteins and proteolysis in pre-term and term human milk and possible implications for infant formulae. International Dairy Journal. 2010; 20:715-723.

[19] Molinari CE, Casadio YS, Hartmann BT, Arthur PG, Hartmann PE. Longitudinal analysis of protein glycosylation and $\beta$-casein phosphorylation in term and preterm human milk during the first 2 months of lactation. British Journal of Nutrition. 2013; 110(1):105-115.

[20] Molinari CE, Casadio YS, Hartmann BT, Livk A, Bringans S, Arthur PG, Hartmann PE. Proteome Mapping of Human Skim Milk Proteins in Term and Preterm Milk. J Proteome Res. 2012; 1:1696-1714.

[21] Bitman J, Wood L, Hamosh M, Hamosh P, Mehta NR. Comparison of the lipid composition of breast milk from mothers of term and preterm infants. The American Journal of Clinical Nutrition. 1983; 38:300-312.

[22] Berenhauser AB. Composição em ácidos graxos do colostro e leite maduro de nutrizes de recém-nascidos prétermo e a termo [dissertação]. Santa Catarina: Universidade Federal de Santa Catarina; 2010.

[23] Fumeaux CJF, Garcia-Rodenas CL, De Castro CC, Courtet-Compondu MC, Thakkar SK et al. Longitudinal Analysis of Macronutrient Composition in Preterm and
Term Human Milk: A Prospective Cohort Study. Nutrients. 2019; 11:1525.

[24] Floris LM, Stahl B, Abrahamse-Berkeveld M, Teller IC. Affiliations expand Human Milk Fatty Acid Profile Across Lactational Stages After Term and Preterm Delivery: A Pooled Data Analysis. Prostaglandins Leukot Essent Fatty Acids. 2020; 156:1-10.

[25] Atkinson SA, Radde IC, Chance GW, Bryan MH, Anderson GH. Macro-mineral content of milk obtained during early lactation from mothers of premature infants. Early Human Development. 1980; 4:5-14.

[26] Sabatier M, Garcia-Rodenas CL, De Castro CA, Kastenmayer P, Vigo M, Dubascoux et al. Longitudinal Changes of Mineral Concentrations in Preterm and Term Human Milk from Lactating Swiss Women. Nutrientes. 2019; 11:1855.

[27] Codo CRB, Caldas JPS, Peixoto RRA, Sanches VL, Guiraldelo TC, Cadore S, Marba STM. Electrolyte and mineral composition of term donor human milk before and after pasteurization and of raw milk of preterm mothers. Revista Paulista de Pediatria. 2018; 36:141-147.

[28] Butte NF, Garza C, Johnson CA, Smith EO, Nichols BL. Longitudinal changes in milk composition of mothers delivering preterm and term infants. Earlv Human Development. 1984; 9:153-162.

[20] Underwood MA. Human Milk for the Premature Infant. Pediatric Clinics of North America. 2013; 60:189207.

[30] Sann L, Bienvenu F, Lahet C, Bienvenu J, Bethenod M. Comparison of the composition of breast milk from mothers of term and preterm infants. Acta Paediatrica Scandinavica. 1981; 70:115-116.

[31] Yamawaki N, Yamada M, Kan-no T, Kojima T, Kaneko T, Yonekubo A. Macronutrient, mineral and trace element composition of breast milk from Japanese women. Journal of Trace Elements in Medicine and Biology. 2005; 19:171-181.

[32] Pishva N, Hemmati F. Comparison of Protein, Fat, Sodium and Calcium in the Milk of Mothers of Preterm and Full term Infants. Shiraz E-Medical Journal. 2004; 5:17 .

[33] Bauer J, Gerss J. Longitudinal analysis of macronutrients and minerals in human milk produced by mothers of preterm infants. European Journal of Clinical Nutrition. 2011; 30:215-220. 
[34] Pessoto MA. Avaliação da lactação em mães de recém-nascido PT com peso de nascimento inferior a 1.250 gramas segundo diferentes métodos de ordenha, manual, com bomba manual ou com bomba elétrica [tese]. Campinas: Universidade Estadual de Campinas; 2009.

[35] Björklund KL, Vahter M, Palm B, Grandér M, Lignell $\mathrm{S}$, Benglund M. Metals and trace element concentrations in breast milk of first time healthy mothers: a biological monitoring study. Environmental Health. 2012; 11:1-8. 